\title{
'Esprit de corps': Towards collaborative integration of pharmacists and nurses into antimicrobial stewardship programmes in South Africa
}

\author{
N Schellack, ${ }^{1}$ BCur, BPharm, PhD; R Pretorius, ${ }^{2}$ BCur, MCur, PhD; A P Messina, ${ }^{3}$ BPharm \\ ${ }^{1}$ Department of Pharmacy, School of Health Care Sciences, Sefako Makgatho Health Sciences University, Pretoria, South Africa \\ ${ }^{2}$ Africa Unit for Transdisciplinary Health Research, Faculty of Health Sciences, North-West University (Potchefstroom Campus), South Africa \\ ${ }^{3}$ Department of Quality Leadership, Netcare Hospitals, Johannesburg, South Africa
}

Corresponding author: N Schellack (natalie.schellack@smu.ac.za)

\begin{abstract}
With the global threat of antimicrobial resistance now more emergent than ever, there should be wider collaboration between members of the multidisciplinary healthcare team. This article proposes possible ways of engagement between the pharmacist, nurse and doctor. The pharmacist and nurse are placed in an ideal position through united efforts (camaraderie) to redirect healthcare towards improved patient outcomes while also reducing antimicrobial resistance.
\end{abstract}

S Afr Med J 2016;106(10):973-974. DOI:10.7196/SAMJ.2016.v106i10.11468

The worldwide spread of antimicrobial resistance (AMR) has been labelled a global health crisis, and it is estimated that by 2050, 10 million lives a year will be at risk as a consequence of antibioticresistant infections, predominantly bacterial. ${ }^{[1]}$ In South Africa (SA), except for tuberculosis, HIV and colistin use in animals, the impact of AMR in the respective sectors is unknown, and a national strategy of utilising all resources to cover more territory to fight AMR is mandatory. The need for wider engagement of healthcare professionals in antimicrobial stewardship programmes (ASPs) is therefore pivotal.

\section{The current situation}

Except for academic centres, in many developing countries it is difficult to replicate resource-abundant stewardship models that are mostly driven by medical microbiology teams and infectious disease (ID) specialists. ${ }^{[2,3]}$ In this regard, effective ASPs depend largely on monitoring practices, teamwork in developing and implementing interventions, and organisational infrastructure. ${ }^{[2]}$ It is therefore critical in the SA context to utilise the existing resources of pharmacists and registered nurses (RNs), who are well placed to co-ordinate anti-infective management and improve patient outcomes. To date, initiatives in SA to promote optimal antimicrobial prescribing have primarily targeted the practices of hospital-based doctors. In addition, until recently very little consideration has been afforded to the contribution nurses can make in anti-infective management and in the development and maintenance of ASPs. This raises the question of how we can broaden involvement of other healthcare professionals in ASPs, build the necessary skills and adapt organisational structures to facilitate effective deployment and integration in the SA national antimicrobial resistance strategy framework $^{[4]}$ and implementation plan. ${ }^{[5]}$

\section{The role of pharmacists and nurses}

Internationally, pharmacists are accepted as equal antimicrobial stewardship (AS) partners. Their focus is to ensure optimal use of antimicrobials and participation or even a leading role in multidisciplinary working groups and committees. Depending on the level of training, the range of antimicrobial management interventions is potentially vast. These include monitoring therapy for appropriateness, providing pharmacokinetic and pharmacodynamic consultation services, in-service training of young doctors, collaborative research into new methods of persuasive interventions, and measuring consumption.

To highlight the indispensable role of pharmacists in recruiting multidisciplinary teams and in co-ordination of interdisciplinary clinician and nurse engagement in AS interventions, Brink et al. ${ }^{[6]}$ recently presented a prospective audit and feedback model for AS in ID-constrained, non-academic settings. This SA study was performed in 47 Netcare hospitals, focusing on basic interventions implemented by pharmacists in consultation with prescribers, and demonstrated a significant reduction in overall antibiotic consumption of $18.1 \%$ in 116662 patients. ${ }^{[6]}$ The Netcare model also confirmed that quality improvement science skills are critical in implementing and maintaining an ASP and that stewardship, dependent on local context and resources, can become embedded in existing systems, which is the key to sustainability. A proposed study of process and outcome measures for the management of communityacquired pneumonia is planned nationally, to promote collaboration and team involvement between both private and public sector pharmacists on how to co-ordinate, implement and monitor ASPs.

As for pharmacists, inclusion in ASPs of the broad-based, multidisciplinary RNs could have a substantial impact. ${ }^{[3]}$ RNs play an essential role in monitoring compliance with institutional guidelines and best practice, monitoring for drug allergies and side-effects, obtaining and reporting of therapeutic levels, management and administration of medicines with mixed dosages, e.g. insulin, and ensuring timely and correct administration of antimicrobials. ${ }^{[7]} \mathrm{A}$ study conducted in 33 Netcare hospitals found that pharmacists and nurses can collectively improve the timeous administration of antibiotics. ${ }^{[8]}$ Among other things, the study involved institutional system changes and the creation of awareness of administration priority, changing perceptions on waiting times for fixed dosing schedules, and adding commonly prescribed antibiotics to ward stock to prevent delays in administration. The improvement in hang-time compliance (antibiotic administration within 1 hour) in 32985 patients following implementation of a process improvement protocol was significant. ${ }^{[8]}$

The decisive role of RNs is exemplified by Brink et al. ${ }^{[9]}$ in a study evaluating improved compliance with four perioperative antibiotic process measures (antibiotic choice, dose, duration and timing) in 34 private SA hospitals. Theatre, anaesthetic and surgical ward RNs assisted 
Table 1. Potential collaborative interventions of pharmacists and registered nurses

\begin{tabular}{ll}
\hline Intervention & Integrative role of registered nurses and pharmacists \\
\hline Antifungal stewardship & $\begin{array}{l}\text { Monitoring the duration and necessity of antifungal therapy following review of } \beta \text {-D-glucan assays and risk scores } \\
(100 \% \text { negative predictivity if both negative })^{[10]}\end{array}$ \\
$\begin{array}{l}\text { Emergency department } \\
\text { stewardship }\end{array}$ & $\begin{array}{l}\text { Actively participate in creating empirical guidelines based on syndromic antibiograms for common community- } \\
\text { acquired infections, ensuring that appropriate cultures are taken, and follow-up of appropriate antimicrobial therapy } \\
\text { after discharge, subsequent to culture sensitivity results }\end{array}$ \\
$\begin{array}{l}\text { Hand hygiene } \\
\text { campaigns }\end{array}$ & $\begin{array}{l}\text { Assisting with independent audit of hand hygiene compliance monitoring as part of hospital strategies for process } \\
\text { improvement and increased awareness of their collective role and responsibility in infection prevention practices }\end{array}$ \\
$\begin{array}{l}\text { Indwelling device } \\
\text { management }\end{array}$ & $\begin{array}{l}\text { Monitoring appropriate device days, changing devices when needed, and excluding indwelling devices as the source of } \\
\text { infection to prevent the irrational use of antimicrobials }\end{array}$ \\
$\begin{array}{l}\text { Therapeutic drug } \\
\text { monitoring }\end{array}$ & $\begin{array}{l}\text { Therapeutic drug monitoring to facilitate effective antimicrobial therapy and timeously identify drug administration } \\
\text { problems, including those involving intravenous administration } \\
\text { Vaccination campaigns }\end{array}$ \\
$\begin{array}{l}\text { Monitoring adherence to vaccination directives and immunisation schedules to reduce mortality and morbidity in } \\
\text { preventable infections }{ }^{[13]}\end{array}$
\end{tabular}

the AS teams in significantly improving compliance with the timely administration of antibiotics prior to surgical incision; a 19.7\% reduction in the surgical site infection rate in 24206 surgical cases from a mean group rate of 2.46 (95\% confidence interval (CI) 2.18 - 2.73) before intervention to 1.97 after intervention ( $95 \%$ CI 1.79 - 2.15) $(p=0.0029)$ was demonstrated. Collectively these local studies illustrate that integrating nurses in ASPs may contribute to a shared sense of responsibility in the care of the patient, increased professional autonomy, and negating the idea that AS is outside the scope of nursing responsibility and expertise. Whereas behavioural changes associated with successful implementation of ASPs among doctors have been studied elsewhere, globally no or very few data for nurses exist. Other potential collaborative interventions in conjunction with AS teams are outlined in Table 1.

\section{Importance of education and training}

The current content and effectiveness of antimicrobial stewardship education of healthcare professionals (HCPs) other than doctors in SA are suboptimal. Furthermore, undergraduate education is variable; a recent study of final-year pharmacy students across SA universities documented significant differences between the eight universities with regard to undergraduate AS education. ${ }^{[14]}$ Furthermore, specialisation in pharmacy at a postgraduate level is not yet formally recognised by the South African Pharmacy Council and not widely available. With regard to nursing, the curriculum currently involves modules on microbiology and pharmacology. However, these are mostly presented as pure rather than applied science, and a new committee to drive change in collaboration with the South African Nursing Council, training institutions and the healthcare industry should be considered.

In addition, information on local nurse practitioners' attitudes, perceptions, and knowledge with regard to antimicrobial use and resistance is mandatory. ${ }^{[15]}$ Local literature on the knowledge and perceptions of nursing practitioners will enable targeted campaigns and short courses focusing on the role of the RN in AS, such as obtaining appropriate specimens for culture, assessing patient response to antimicrobial therapy, and decreasing hospital-acquired infections through the implementation of care bundles, thus increasing awareness and vigilance among RNs during the administration of antimicrobials. RNs also need to contribute to research in the field by investigating strategies that involve and define nurse involvement and ascertain the impact they could have on antimicrobial utilisation and infection prevention beyond hand hygiene. This would be of particular relevance to community RNs and retail pharmacists from an outpatient or primary care viewpoint; to date, few stewardship interventions have targeted general practitioners and non-hospital-based HCPs in SA.
Towards the future of integrated teams Pharmacists and RNs are well positioned to actively collaborate in AS teams and substantially contribute to optimal antimicrobial management in acute, ward and outpatient settings. However, given the absence of institutional ID resources in most SA hospitals, recruitment and active participation of more prescribers such as surgeons, intensive care physicians, paediatricians and others in such teams are necessary. For such initiatives to positively and sustainably impact on outcomes, it is therefore necessary to gain a contextual understanding of the barriers and facilitators to pharmacists and nurses contributing to AS, and how this role may be developed in the future. 'Esprit de corps', a French term referring to a shared spirit of comradeship, enthusiasm and devotion to a cause among the members of a group, reflects such a united front to tackle antimicrobial resistance in SA. Recognition and acknowledgment of the potential role of HCPs other than doctors are critical to ensure sustainable antimicrobial preservation.

\footnotetext{
1. O Neill J. The Review on Antimicrobial Resistance. Final report. http://amr-review.org/Publications (accessed 18 August 2016).

2. Charani E, Edwards R, Sevdalis $\mathrm{N}$, et al. Behaviour change strategies to influence antimicrobial prescribing in acute care: A systematic review. Clin Infect Dis 2011;53(7):651-662. DOI:10.1093/cid/cit212

3. Olans RN, Olans RD, DeMaria A. The critical role of the staff nurse in antimicrobial stewardship unrecognized, but already there. Clin Infect Dis 2016;62(1):84-89. DOI:10.1093/cid/civ697

4. National Department of Health, South Africa. Antimicrobial Resistance National Strategy Framework 4. National Department of Health, South Africa. Antimicrobial Resistance National Strategy Framework
2014-2024. http://www.health.gov.za/index.php/antimicrobial-resistance (accessed 14 August 2016). 5. National Department of Health, South Africa. Implementation Plan for Antimicrobial Resistance National Strategy 5. National Department of Health, South Africa. Implementation Plan for Antimicrobial Resistance National Stra
Framework 2014-2019. http://www.health.govza/index.php/antimicrobial-resistance (accessed 20 July 2016).

Framework 2014-2019. http://wwwhealth.gov:za/index.php/antimicrobial-resistance (accessed 20 July 2016).
6. Brink AJ, Messina AP, Feldman C, et al., on behalf of the Netcare Antimicrobial Stewardship Study 6. Brink AJ, Messina AP, Feldman C, et al., on behalf of the Netcare Antimicrobial Stewardship Study
Alliance. Antimicrobial stewardship across 47 South African hospitals: An implementation study. Lancet Infect Dis 2016;16(9):1017-1025. DOI:10.1016/S1473-3099(16)30012-3

7. Edwards R, Drumright LN, Kiernan M, Holmes A. Covering more territory to fight resistance: Considering nurses' role in antimicrobial stewardship. J Infect Prev 2011;12(1):6-10. DOI:10.1177/1757177410389627

8. Messina AP, van den Bergh D, Goff DA. Antimicrobial stewardship with pharmacist intervention improves timeliness of antimicrobials across thirty-three hospitals in South Africa. Infect Dis Ther 2015;4(Suppl 1):5-14. DOI:10.1007/s40121-015-0082-x

9. Brink AJ, Messina AP, Feldman C, et al. From guidelines to practice: A pharmacist-driven prospective audit and feedback improvement model for peri-operative antibiotic prophylaxis in 34 South African hospitals. J Antimicrob Chemother 2016 (in press).

10. Posteraro B, Tumbarello M, de Pascale G, et al. (1,3)- $\beta$-D-glucan-based antifungal treatment in critically ill adults at high risk of candidaemia: An observational study. J Antimicrob Chemother 2016;71(8):2262-2269. DOI:10.1093/jac/dkw112

11. Pulcini C. Antimicrobial stewardship in emergency departments: A neglected topic. Emerg Med J 2015;32:506. DOI:10.1136/emermed-2014-204220

12. Mendelson M, Matsotso MP. A global call for action to combat antimicrobial resistance: Can we get it right this time? S Afr Med J 2014;104(7):478-479. DOI:10.7196/SAMJ.8534

13. Fong DJ. Immunizations: Demonstrating the value of the community pharmacist and enabling HIT progress. 2016. http://www.wolterskluwercdi.com/blog/immunizations-demonstrating-valuecommunity-pharmacist-and-enabling-hit-progress/ (accessed 27 July 2016).

14. Burger M, Fourie J, Loots D, et al. Knowledge and perceptions of antimicrobial stewardship concepts among final year pharmacy students in pharmacy schools across South Africa. South Afr J Infect Dis 2016;1(1):1-7. DOI:10.1080/23120053.2016.1192808

15. Abbo L, Smith L, Pereyra M, et al. Nurse practitioners' attitudes, perceptions, and knowledge about antimicrobial stewardship. J Nurse Pract 2012;8(5):370-376. DOI:10.1016/j.nurpra.2012.01.023
} 